\title{
microRNA-802 promotes lung carcinoma proliferation by targeting the tumor suppressor menin
}

\author{
LUN-QING WANG $^{1,2}$, GANG CHEN ${ }^{1}$, XIANG-YAN LIU ${ }^{1}$, FAN-YING LIU ${ }^{1}$, SHAO-YAN JIANG ${ }^{3}$ and ZHOU WANG $^{1}$ \\ ${ }^{1}$ Department of Thoracic Surgery, Provincial Hospital Affiliated to Shandong University, Jinan, Shandong 250021; \\ ${ }^{2}$ Department of Thoracic Surgery, The East Part of Qingdao Municipal Hospital, Qingdao, Shandong 266071; \\ ${ }^{3}$ Department of Cardiology, The Affiliated Cardiovascular Hospital of Medical College, \\ Qingdao University, Jinan, Shandong 266071, P.R. China
}

Received August 29, 2013; Accepted May 6, 2014

DOI: $10.3892 / \mathrm{mmr} .2014 .2361$

\begin{abstract}
RNAs play important roles in numerous biological processes, including tumorigenesis, by modulating critical gene transcripts. In the present study, the role of microRNA-802 (miR-802) in lung cancer was investigated. The results of the quantitative polymerase chain reaction revealed that expression levels of miR-802 were significantly upregulated in lung cancer tissues. In vitro experiments demonstrated that miR-802 promoted cell proliferation in A549, NCI-H358 and NCI-H1299 cells. Furthermore, it was indicated that miR-802 promoted the proliferation of lung carcinoma by targeting the tumor suppressor menin. Therefore, these results suggest a previously unknown miR-802/menin molecular network controlling lung carcinoma development.
\end{abstract}

\section{Introduction}

Lung cancer, including small cell lung cancer and non-small cell lung cancer, has become the most common cause of cancer-related mortality worldwide (1). Therefore, understanding the mechanisms of lung cancer for the development of effective therapies is of great importance.

microRNAs (miRNAs), a class of noncoding RNAs, between 19 and 30 nucleotides in length, post-transcriptionally modulate gene expression by negatively regulating the stability or translational efficiency of certain mRNAs $(2,3)$. Accumulating evidence has shown that the miRNAs have critical roles in numerous processes, including developmental events, cell differentiation and apoptosis, glucose and lipid metabolism and cancer initiation and progression $(4,5)$. Furthermore, a number of recent studies have found that the dysregulated expression of certain miRNAs is involved in lung cancer and that this contrib-

Correspondence to: Dr Zhou Wang, Department of Thoracic Surgery, Provincial Hospital Affiliated to Shandong University, 324 Jingwu Weiqi Road, Jinan, Shandong 250021, P.R. China E-mail:wz620226@yeah.net

Key words: lung carcinoma, microRNA, miR-802, menin utes to tumorigenesis (6-8). miR-143, for example, has been found to be downregulated in lung cancer tissues and cell lines. It has been demonstrated that, at the molecular level, miR-143 significantly inhibits the migration and invasion of lung cancer cells by targeting cluster of differentiation $44 \mathrm{v} 3$ (9). Therefore, the identification and understanding of the specific roles of certain miRNAs may provide a novel and promising strategy for the development of therapeutic treatments against cancer.

In the present study, miR-802 was investigated as a positive regulator of proliferation in lung cancer cell lines (A549, NCI-H358 and NCI-H1299). It was hypothesized that miR-143 may regulate proliferation by targeting the tumor suppressor menin.

\section{Material and methods}

Tissue samples. A total of 40 pairs of tumor tissues and adjacent normal tissues were collected from routine therapeutic surgery at the Department of Thoracic Surgery, Qingdao Municipal Hospital (Qingdao, China). All samples were obtained with informed consent and approved by the Qingdao Municipal Hospital institutional review board.

Cell culture. Three lung cancer cell lines (A549, NCI-H358 and NCI-H1299) were obtained from the Chinese Academy of Sciences Cell Bank (Shanghai, China). Cells were cultured in Dulbecco's modified Eagle's medium (Gibco-BRL, Beijing, China) supplemented with $10 \%$ fetal bovine serum (Gibco-BRL). Cultures were maintained at $37^{\circ} \mathrm{C}$ in a humidified atmosphere with $5 \% \mathrm{CO}_{2}$.

Analysis of miRNA expression using TaqMan ${ }^{\circledR}$ quantitative polymerase chain reaction ( $q P C R$ ). Total RNA from tissues or cell lines was harvested using the miRNA Isolation kit (Ambion ${ }^{\circledR}$, Austin, TX, USA). The expression of mature miRNAs was assayed using the TaqMan miRNA Assay (Applied Biosystems ${ }^{\mathrm{TM}}$, Foster City, CA, USA) specific for hsa-miR-802. Briefly, 5 ng total RNA was reverse transcribed to cDNA using specific stem-loop reverse transcription primers. qPCR was performed using an Applied Biosystems 7500 Real-Time PCR System and a TaqMan Universal PCR Master Mix. All the primers were obtained from the TaqMan miRNA 
Assays. U6 small nuclear RNA (Applied Biosystems) was used as an internal control.

BrdU assays. A cell proliferation ELISA (BrdU kit; Beyotime, Haimen, China) was used to analyze the incorporation of BrdU during DNA synthesis in accordance with the manufacturer's instructions. All experiments were performed in triplicate. Absorbance was measured at $450 \mathrm{~nm}$ in the Spectra Max 190 ELISA reader (Molecular Devices, Sunnyvale, CA, USA)

Plasmid construction and transfection. For the miR-802 expression plasmid, human miR-802 precursor was cloned into pSilencer 4.1 (Ambion). The negative control plasmid consisted of a scrambled sequence (Ambion). To inhibit miR-802 function, an Ambion miRNA inhibitor of miR-802 was used, along with the negative control. For transfection, a complex of Lipofectamine 2000 (Invitrogen Life Technologies, Carlsbad, CA, USA) and $25 \mathrm{nM}$ of the miRNAs mentioned above was prepared in accordance with the manufacturer's instructions.

miR-802 targets prediction. The putative targets of miR-802 were predicted using the miRWalk software (10). The algorithm produced a list of hundreds of target genes for miR- 802 by searching for the presence of conserved heptamer and octamer sites matching the seed region of miR-802.

Western blot analysis. Cells were harvested and lysed with ice-cold lysis buffer (50 mM Tris-HCl, pH 6.8, 2 mM 2-mercaptoethanol, $2 \% \mathrm{w} / \mathrm{v}$ SDS and $10 \%$ glycerol). The cells were centrifuged at $20,000 \mathrm{x}$ g for $10 \mathrm{~min}$ at $4^{\circ} \mathrm{C}$, and the proteins in the supernatant were quantified and separated using 10\% SDS PAGE and transferred to a nitrocellulose membrane (Amersham Pharmacia Biotech, Amersham, UK). Following blocking with $10 \%$ nonfat milk in phosphate-buffered saline, membranes were immunoblotted with antibodies as indicated, followed by horseradish peroxidase-linked secondary antibodies (Cell Signaling Technology, Inc., Beverly, MA, USA). The signals were detected using a SuperSignal ${ }^{\mathrm{TM}}$ West Pico Chemiluminescent Substrate kit (Pierce, Rockford, IL, USA) in accordance with manufacturer's instructions. Anti-menin, -p18 and -p27 antibodies were purchased from Abcam (Cambridge, MA, USA). Anti- $\beta$-catenin and -p65 antibodies were purchased from Cell Signaling Technology, Inc. Protein levels were normalized against GAPDH or Lamin B (Santa Cruz Biotechnology, Inc., Santa Cruz, CA, USA).

Luciferase reporter assay. Total cDNA from A549 cells was used to amplify the 3'-untranslated region (UTR) of menin by PCR. The menin 3'-UTR was cloned into pMIR-REPORT TM (Ambion), resulting in pMIR-REPORT-menin. Mutations were introduced into potential miR-802 binding sites using the QuikChange ${ }^{\circledR}$ Site-Directed Mutagenesis kit (Stratagene, La Jolla, CA, USA). Cells were transfected with the pMIR-REPORT vectors containing the 3 '-UTR variants, the miR- 802 precursor and control plasmids for $36 \mathrm{~h}$. The pRL-TK vector (Promega Corporation, Madison, WI, USA) carrying the Renilla luciferase gene was used as an internal control to normalize the transfection efficiency. Luciferase values were determined using the Dual-Luciferase Reporter Assay system (Promega Corporation).

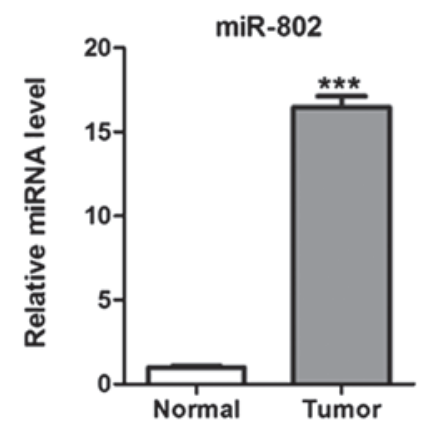

Figure 1. Expression levels of miR-802 in lung cancer tissues. miR-802 expression was determined using a quantitative polymerase chain reaction in human lung cancer tissues (Tumor) and adjacent noncancerous tissues (Normal). ${ }^{* * *} \mathrm{P}<0.001$ compared with normal tissues. miR-802, microRNA-802.

Flow cytometric analysis. To assess the distribution of nuclear DNA content, cells were collected, rinsed and fixed overnight in $75 \%$ cold ethanol at $-20^{\circ} \mathrm{C}$. Subsequently, cells were treated with Tris- $\mathrm{HCl}$ buffer (pH 7.4) supplemented with $100 \mu \mathrm{g} / \mathrm{ml}$ RNase A and stained with $25 \mu \mathrm{g} / \mathrm{ml}$ propidium iodide (BD Biosciences, San Diego, CA, USA). Cell cycle distribution was determined by flow cytometry (Becton Dickinson, San Jose, CA, USA). In total, 10,000 cells were acquired and analyzed for DNA content. All data were collected, stored and analyzed by ModFit software (Becton Dickinson).

Statistical analysis. Data are expressed as the mean \pm standard error of the mean from at least three separate experiments. Differences between groups were analyzed using a Student's t-test analysis. $\mathrm{P}<0.05$ was considered to indicate a statistically significant difference.

\section{Results}

miR-802 expression levels are increased in lung carcinoma tissues. miR-802 expression levels were determined using TaqMan qPCR in 40 pairs of human lung carcinoma tissues and adjacent matched noncancerous tissues. As shown in Fig. 1, the expression levels of miR-802 were significantly upregulated in cancer tissues in comparison with those in adjacent noncancerous tissues.

miR-802 overexpression promotes lung cancer cell proliferation. To determine the effects of miR-802 on lung carcinoma cell growth, miR-802 precursor was transfected into A549, NCI-H358 and NCI-H1299 cells (Fig. 2A-C). As a result of miR-802 overexpression, the proliferative ability in these cells post-transfection was significantly enhanced (Fig. 2D-F). Furthermore, cells overexpressing miR-802 had a significantly lower percentage of cells in the G1/G0 phase and increased percentage of cells in the $\mathrm{S}$ phase, compared with negative control-transfected cells (Fig. 2G-I).

miR-802 antisense inhibits the proliferation of lung cancer cells. Cells were transfected with miR-802 antisense to block the functions of endogenous miR-802. As a result of ectopic expression of the hsa-miR-802 antisense, a reduction in proliferative ability was observed in A549, NCI-H358 and 
A

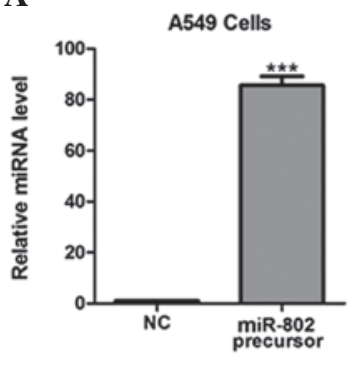

D

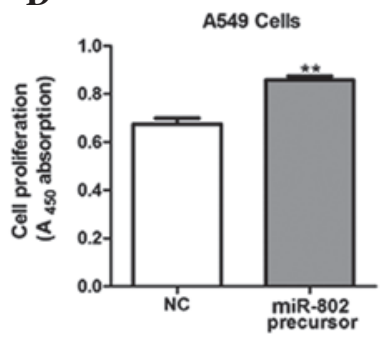

G

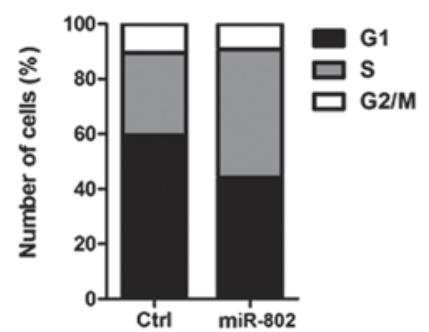

B

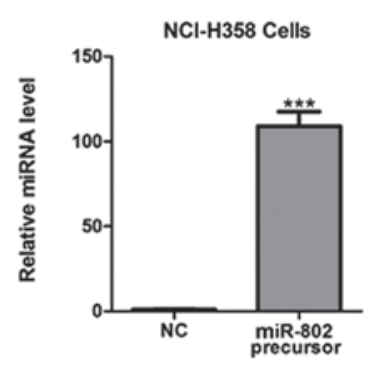

E

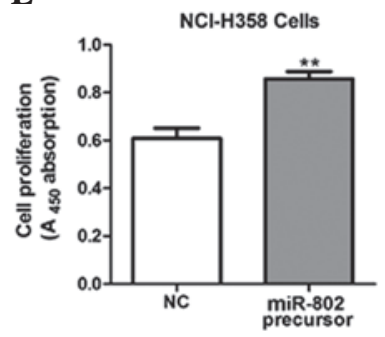

H

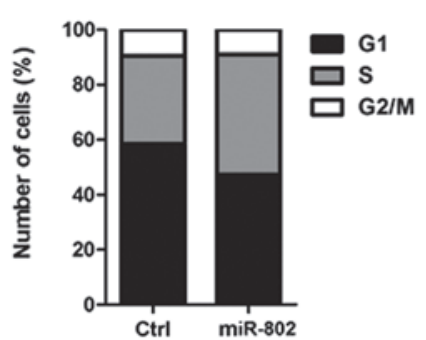

C

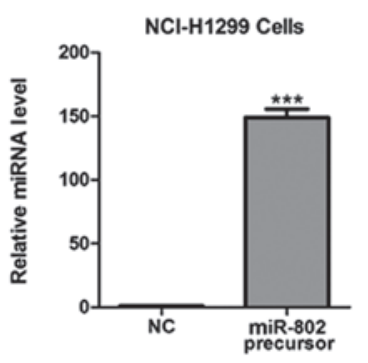

F

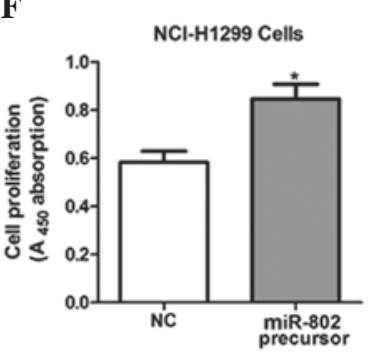

I

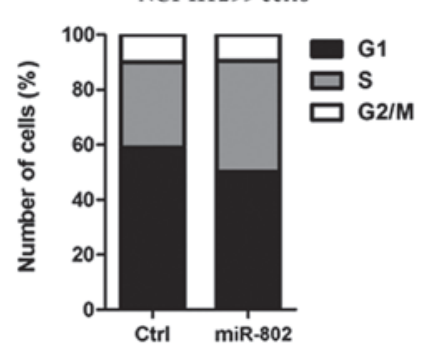

Figure 2. Overexpression of miR-802 promotes lung cancer cell proliferation. (A-C) Expression of miR-802 was determined in (A) A549, (B) NCI-H358 and (C) NCI-H1299 cells transfected with miR-802 precursor or NC plasmids. ${ }^{* * *} \mathrm{P}<0.001$ compared with NC. (D-F) Cell proliferation (BrdU) was determined in (D) A549, (E) NCI-H358 and (F) NCI-H1299 cells transfected with miR-802 precursor or NC plasmids. $\mathrm{A}_{450}$ absorption was assayed $24 \mathrm{~h}$ after transfection. ${ }^{*} \mathrm{P}<0.05$ and ${ }^{* *} \mathrm{P}<0.01$ compared with NC. (G-I) Cell cycle analysis of (G) A549, (H) NCI-H358 and (I) NCI-H1299 cells transfected with miR-802 precursor or NC plasmids using flow cytometry. Cells were labeled for $15 \mathrm{~min}$ with propidium iodide and immediately analyzed using flow cytometry. Bar charts represent the percentage of cells in each phase of the cell cycle (G0/G1, S and G2/M). miR-802, microRNA-802; Ctrl, control; NC, negative control.

A

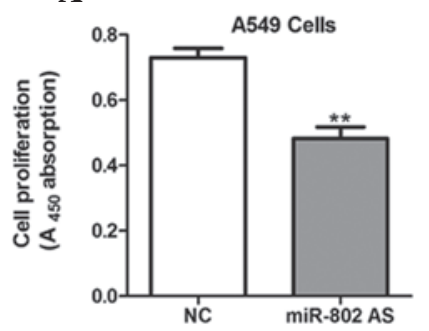

D

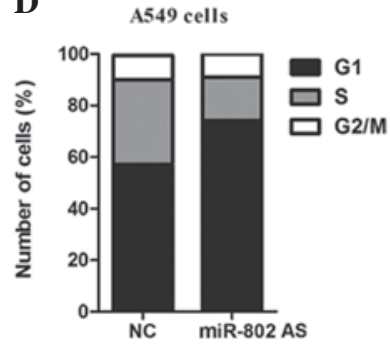

B

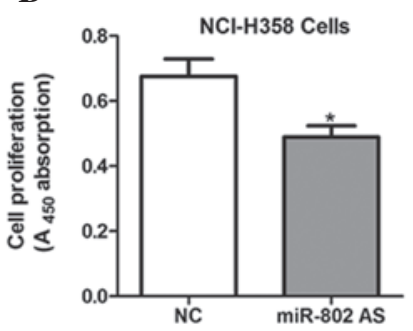

$\mathbf{E}$

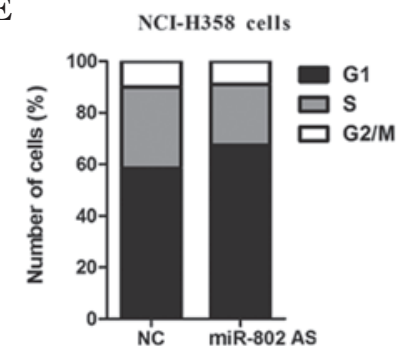

C

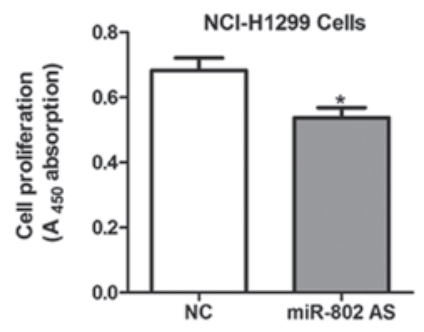

F

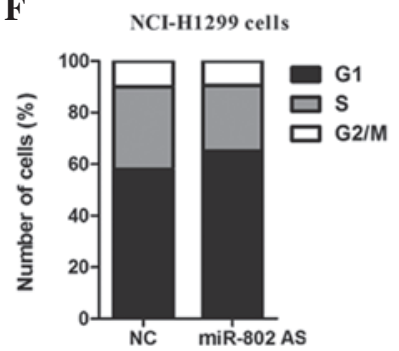

Figure 3. miR-802 AS inhibits the proliferation of lung cancer cells. (A-C) Cell proliferation (BrdU) was determined in (A) A549, (B) NCI-H358 and (C) NCI-H1299 cells transfected with miR-802 AS or NC plasmids. $\mathrm{A}_{450}$ absorption was assayed $24 \mathrm{~h}$ after transfection. ${ }^{*} \mathrm{P}<0.05$ and ${ }^{* *} \mathrm{P}<0.01 \mathrm{compared}$ with NC. (D-F) Cell cycle analysis of (D) A549, (E) NCI-H358 and (F) NCI-H1299 cells transfected with miR-802 AS or NC plasmids using flow cytometry. miR-802, microRNA-802; AS, antisense; NC, negative control. 
A

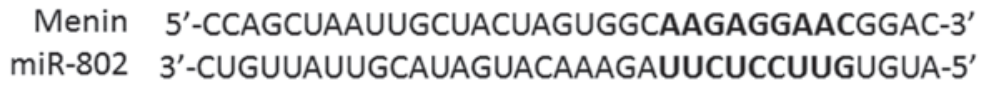

B

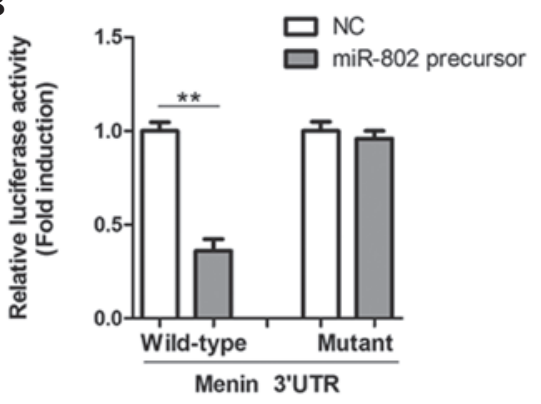

D

$$
\text { A549 Cells }
$$

NC miR-802 AS

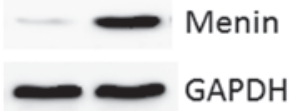

C

\author{
A549 Cells \\ NC $\underset{\text { precursor }}{\operatorname{miR}-802}$
}

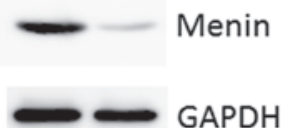

$\mathbf{E}$

$\mathrm{NCl}-\mathrm{H} 358$ Cells

NC $\underset{\text { precurso }}{\operatorname{miR}-802}$

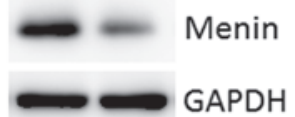

F

\author{
$\mathrm{NCl}-\mathrm{H} 358$ Cells \\ NC miR-802 AS
}

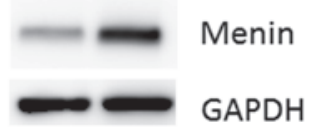

G NCl-H1299 Cells

NC $\begin{gathered}\text { miR-802 } \\ \text { precursor }\end{gathered}$

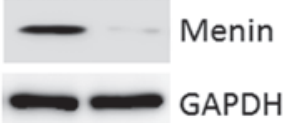

H NCl-H1299 Cells

NC miR-802 AS

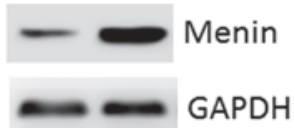

Figure 4. miR-802 represses menin expression by targeting its 3'-UTR. (A) Prediction of miR-802 binding sites in the 3'-UTRs of the human menin gene using miRWalk software. Potential binding sites are highlighted in bold. (B) Luciferase reporter assays in A549 cells. Cells were transfected with 200 ng wildtype or mutant 3'-UTR-reporter constructs together with $50 \mathrm{nM}$ miR-802 precursor or NC. (C-D) Western blot analysis of menin protein expression in A549 cells transfected with (C) miR-802 precursor, (D) miR-802 AS or NC. (E-H) Western blot analysis of menin protein expression in (E and F) NCI-H358 and (G and H) NCI-H1299 cells transfected with (E and G) miR-802 precursor, (F and H) miR-802 AS or NC. miR-802, microRNA-802; UTR, untranslated region, AS, antisense; NC, negative control.

A

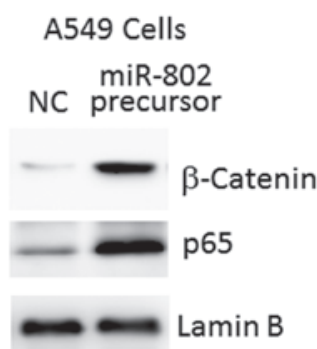

D

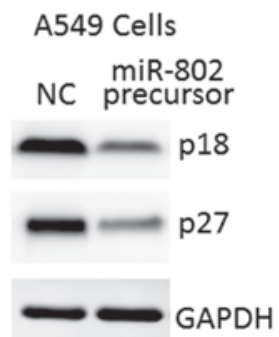

B NCl-H358 Cells

miR-802

NC precursor

$\beta$-Catenin

p65

Lamin B

E

$\mathrm{NCl}-\mathrm{H} 358$ Cells
miR-802

NC precursor

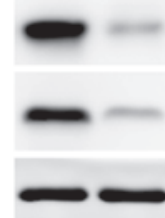

p18

p27

GAPDH
C NCl-H1299 Cells miR-802

NC precursor

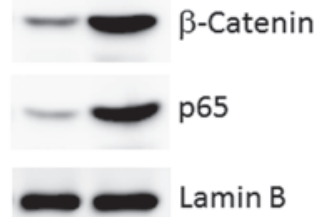

F

$\mathrm{NCl}-\mathrm{H} 1299$ Cells

NC $\begin{gathered}\text { miR-802 } \\ \text { precursor }\end{gathered}$
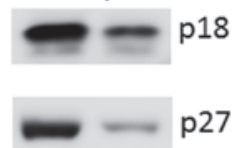

p27

GAPDH

Figure 5. miR-802 regulates downstream signaling and gene expression of menin. (A-C) Nuclear $\beta$-catenin and p65 expression was determined using western blot analysis in (A) A549, (B) NCI-H358 and (C) NCI-H1299 cells transfected with miR-802 precursor or NC. Lamin B level was used as a loading control. (D-F) p18 and p27 expression levels were determined using western blot analysis in (D) A549, (E) NCI-H358 and (F) NCI-H1299 cells transfected with miR-802 precursor or NC. GAPDH level was used as a loading control. miR-802, microRNA-802; NC, negative control. 
NCI-H1299 cells compared with negative control-transfected cells (Fig. 3A-C). Additionally, inhibition of miR-802 significantly increased the percentage of cells in the G0/G1 phase and decreased the percentage of cells in the S phase (Fig. 3D-F).

miR-802 targets the tumor suppressor menin and downregulates its expression. Using a stringent bioinformatics approach (miRWalk software), several putative human miR-802 target genes were identified (data not shown), including the tumor suppressor gene menin (Fig. 4A). To investigate whether miR-802 was able to directly bind to the 3'-UTR of menin and repress its expression, luciferase report vectors containing the putative miR-802 binding sites within the menin 3'-UTR were constructed. As shown in Fig. 4B, overexpression of miR-802 led to a reduction in luciferase activity when the reporter construct contained the menin 3'-UTR in A549 cells. To confirm that menin was a direct target of miR-802, further luciferase reporter assays were performed with vectors containing a mutant menin 3'-UTR. The mutation in the miR-802 binding site from the menin 3'-UTR abolished this effect of miR-802, indicating that miR-802 directly inhibits menin expression by targeting its 3'-UTR (Fig. 4B).

The protein expression levels of menin showed an inverse correlation with miR-802 expression in A549 cells. miR-802 precursor inhibited menin expression while miR-802 antisense increased menin protein expression, as indicated by the results from the western blot analysis (Fig. 4C and D). Similar results were also observed in NCI-H358 and NCI-H1299 cells (Fig. 4E-H).

miR-802 regulates downstream signaling of the tumor suppressor menin. A reduction in menin expression or activity has been found to lead to increased $\mathrm{Wnt} / \beta$-catenin and nuclear factor $\kappa$-light-chain-enhancer of activated B cells $(\mathrm{NF}-\kappa \mathrm{B}) / \mathrm{p} 65$ activation in numerous types of tumor tissues and cells $(11,12)$. As shown in Fig. 5A and B, overexpression of miR-802 led to activation of $\mathrm{Wnt} / \beta$-catenin and $\mathrm{NF}-\kappa \mathrm{B} / \mathrm{p} 65$ signaling in lung carcinoma cells, as demonstrated by the increased levels of nuclear $\beta$-catenin and p65 protein (Fig. 5A-C).

By contrast, menin has been found to upregulate expression of p18 and p27, two negative regulators of cell cycle progression, through recruitment of the histone methyltransferase protein complex (13). In accordance with this, reduced expression of p18 and p27 in lung carcinoma cells overexpressing miR-802 was also observed in the present study (Fig. 5D-F). Therefore, these results further indicate that menin is an important target gene of miR-802 in lung carcinoma cells.

\section{Discussion}

In the present study, miR-802 was characterized as an miRNA that has an important role in the development of lung carcinoma. A previous study demonstrated that miR-802 modulates the biological efficacy of angiotensin II (Ang II) in the human gastrointestinal tract through regulation of the Ang II type 1 receptor expression (14). Furthermore, Kornfeld et al (15) demonstrated that the deregulated expression of miR-802 has a critical role in the development of the obesity-associated impairment of glucose metabolism through targeting of hepatocyte nuclear factor-1 $\beta$ (14). However, the present study is the first, to the best of our knowledge, to demonstrate that miR-802 is expressed in lung cancer tissues, which suggests that miR-802 may act as an oncogene in lung cancer.

To verify this hypothesis, the functional roles of miR-802 in three lung cancer cell lines were investigated. Using cell viability assays and cell cycle analysis it was demonstrated that selective overexpression of miR-802 enhanced cell proliferation, whilst inhibition of miR-802 repressed cell proliferation. Furthermore, using the luciferase reporter system assay in A549 cells, menin was identified as a novel direct target of miR-802. In addition, western blot analysis confirmed that menin and its downstream target genes were regulated by miR-802.

A previous study found that menin may be the pathogenic gene for insulinoma in patients with multiple endocrine neoplasia type 1 (16). Menin has also been identified as a tumor suppressor in other types of tumors, including pituitary and parathyroid tumors, as well as adrenocortical and lung carcinoma (17). In addition, gene mutations in menin have been found in sporadic carcinoid tumors of the lung $(18,19)$. At the molecular level, menin negatively regulates A549 cell proliferation and invasion, which is mediated by the growth factor pleiotrophin and its cell surface receptor, protein tyrosine phosphatase $\beta / \zeta(20,21)$.

The present study confirms that miR-802 is upregulated in lung cancer tissues. For the first time, to the best of our knowledge, miR-802 has been demonstrated to significantly promote the proliferation of lung cancer cells. Menin was also identified as a novel target gene involved in miR-802-mediated cell proliferation in lung cancer. These results indicate that miR-802 may have an important role in lung cancer, and suggest that inhibition of miR-802 may be a potential therapeutic strategy for the treatment of lung cancer.

\section{References}

1. Keith RL and Miller YE: Lung cancer chemoprevention: current status and future prospects. Nat Rev Clin Oncol 10: 334-343, 2013.

2. Kim VN, Han J and Siomi MC: Biogenesis of small RNAs in animals. Nat Rev Mol Cell Biol 10: 126-139, 2009.

3. Ameres SL and Zamore PD: Diversifying microRNA sequence and function. Nat Rev Mol Cell Biol 14: 475-488, 2013.

4. van Kouwenhove M, Kedde M and Agami R: MicroRNA regulation by RNA-binding proteins and its implications for cancer. Nat Rev Cancer 11: 644-656, 2011.

5. Kasinski AL and Slack FJ: Epigenetics and genetics. MicroRNAs en route to the clinic: progress in validating and targeting microRNAs for cancer therapy. Nat Rev Cancer 11: 849-864, 2011.

6. Nana-Sinkam SP and Croce CM: Clinical applications for microRNAs in cancer. Clin Pharmacol Ther 93: 98-104, 2013.

7. Sittka A and Schmeck B: MicroRNAs in the lung. Adv Exp Med Biol 774: 121-134, 2013

8. Redova M, Sana J and Slaby O: Circulating miRNAs as new blood-based biomarkers for solid cancers. Future Oncol 9: 387-402, 2013.

9. Ma Q, Jiang Q, Pu Q, Zhang X, Yang W, et al: MicroRNA-143 inhibits migration and invasion of human non-small-cell lung cancer and its relative mechanism. Int J Biol Sci 9: 680-692, 2013.

10. Dweep H, Sticht C, Pandey P and Gretz N: miRWalk - database: prediction of possible miRNA binding sites by 'walking' the genes of 3 genomes. J Biomed Inform 44: 839-847, 2011.

11. Seigne C, Auret M, Treilleux I, Bonnavion R, Assade F, et al: High incidence of mammary intraepithelial neoplasia development in Men1-disrupted murine mammary glands. J Pathol 229: 546-558, 2013. 
12. Gang D, Hongwei H, Hedai L, Ming Z, Qian H and Zhijun L: The tumor suppressor protein menin inhibits NF- $\kappa \mathrm{B}$-mediated transactivation through recruitment of Sirt1 in hepatocellular carcinoma. Mol Biol Rep 40: 2461-2466, 2013.

13. Veniaminova NA, Hayes MM, Varney JM and Merchant JL: Conditional deletion of menin results in antral $\mathrm{G}$ cell hyperplasia and hypergastrinemia. Am J Physiol Gastrointest Liver Physiol 303: G752- G764, 2012.

14. Sansom SE, Nuovo GJ, Martin MM, Kotha SR, Parinandi NL and Elton TS: miR-802 regulates human angiotensin II type 1 receptor expression in intestinal epithelial C2BBel cells. Am J Physiol Gastrointest Liver Physiol 299: G632- G642, 2010.

15. Kornfeld JW, Baitzel C, Könner AC, Nicholls HT, Vogt MC, et al: Obesity-induced overexpression of miR-802 impairs glucose metabolism through silencing of Hnf1b. Nature 494: 111-115, 2013.

16. Gaztambide $S$, Vazquez $F$ and Castaño L: Diagnosis and treatment of multiple endocrine neoplasia type 1 (MEN1). Minerva Endocrinol 38: 17-28, 2013.
17. Matkar S, Thiel A and Hua X: Menin: a scaffold protein that controls gene expression and cell signaling. Trends Biochem Sci 38: 394-402, 2013

18. Debelenko LV, Brambilla E, Agarwal SK, Swalwell JI, Kester MB, et al: Identification of MEN1 gene mutations in sporadic carcinoid tumors of the lung. Hum Mol Genet 6: 2285-2290, 1997.

19. Haruki N, Yatabe Y, Travis WD, Nomoto S, Osada H, et al: Characterization of high-grade neuroendocrine tumors of the lung in relation to menin mutations. Jpn J Cancer Res 91: 317-323, 2000.

20. Gao SB, Feng ZJ, Xu B, Wu Y, Yin P, et al: Suppression of lung adenocarcinoma through menin and polycomb gene-mediated repression of growth factor pleiotrophin. Oncogene 28: 4095-4104, 2009.

21. Feng ZJ, Gao SB, Wu Y, Xu XF, Hua X and Jin GH: Lung cancer cell migration is regulated via repressing growth factor PTN/RPTP $\beta / \zeta$ signaling by menin. Oncogene 29: 5416-5426, 2010. 